TRANSACTIONS OF THE

AMERICAN MATHEMATICAL SOCIETY

Volume 363, Number 10, October 2011, Pages 5091-5109

S 0002-9947(2011)05138-X

Article electronically published on May 11, 2011

\title{
TUBE STRUCTURES, HARDY SPACES AND EXTENSION OF CR DISTRIBUTIONS
}

\author{
G. HOEPFNER, J. HOUNIE, AND L. A. CARVALHO DOS SANTOS
}

\begin{abstract}
We consider rough tubes $X+i \mathbb{R}^{m} \subset \mathbb{C}^{m}$, where $X \subset \mathbb{R}^{m}$ is a measurable set, and extend the notion of $C R$ function to the space $L^{\infty}\left(X, h^{p}\left(\mathbb{R}^{m}\right)\right)$, where $h^{p}\left(\mathbb{R}^{m}\right), 0<p<\infty$, is Goldberg's semilocal Hardy space. We show that if $X$ is the image of some connected manifold by some $C^{1}$ map, then all such $C R$ functions can be extended to the convex hull of the tube as $C R$ functions $\in L^{\infty}\left(\operatorname{ch}(X), h^{p}\left(\mathbb{R}^{m}\right)\right)$. This extends previous work of Boggess.
\end{abstract}

\section{INTRODUCTION}

The classical Bochner tube theorem ( $[\overline{\mathrm{BO}}, \overline{\mathrm{BM}}]$ ) states that if $\Omega \subset \mathbb{R}^{m}$ is a connected open set, any holomorphic function $f(z)$ defined on the tube $\Omega+i \mathbb{R}^{m} \subset$ $\mathbb{C}^{m}$ can be holomorphically extended to the convex hull $\operatorname{ch}(\Omega)+i \mathbb{R}^{m}$. This theorem has been extended by Boivin and Dwilewicz $\mathrm{BD}$ to tubes $N+i \mathbb{R}^{m}$, where $N \subset$ $\mathbb{R}^{m}$ is a connected embedded submanifold, by showing that any continuous CR function on $N+i \mathbb{R}^{m}$ can be extended to a continuous CR function on the interior of $\operatorname{ch}(N)+i \mathbb{R}^{m}$ (where the interior is taken with respect to the affine subspace generated by $\operatorname{ch}(N))$.

It is natural to consider CR functions on tubes that have restricted growth at infinity in the tube direction, and Boggess [B2] studied the case of CR functions on tubes $N+i \mathbb{R}^{m}$ ( $N$ a smooth, connected, embedded submanifold of $\mathbb{R}^{m}$ ) that satisfy a uniform bound on the $L^{p}$ norm in the tube direction, $1 \leq p \leq \infty$. He proved an extension theorem to the interior of the convex hull of $N+i \mathbb{R}^{m}$ (here the interior is taken in $\mathbb{R}^{m}$ ) preserving the $L^{p}$ bounds, which leads to a theory of Hardy spaces on tubes.

In this work we extend Boggess' results in several ways; in particular, we consider rough tubes $X+i \mathbb{R}^{m}$, where $X \subset \mathbb{R}^{m}$ is a measurable set and we let $p$ take values in the range $0<p<\infty$. We work within the framework of the semilocal Hardy spaces $h^{p}\left(\mathbb{R}^{m}\right)$ introduced by Goldberg $\left[\mathrm{G}\right.$, which are subspaces of the space $\mathcal{S}^{\prime}\left(\mathbb{R}^{m}\right)$ of tempered distributions for $0<p \leq \infty$ and for $p>1$ coincide with the Lebesgue spaces $L^{p}\left(\mathbb{R}^{m}\right)$. To deal with rough tubes we generalize the notion of $\mathrm{CR}$ function as follows: a function $F \in L^{\infty}\left(X, h^{p}\left(\mathbb{R}^{m}\right)\right)$ is a (generalized) CR function if there exists a sequence $F_{j}(x+i y)$ of entire functions in $\mathbb{C}^{m}$ such that for some $C>0$,

Received by the editors May 11, 2009.

2000 Mathematics Subject Classification. Primary 32A35, 32V25, 35N10; Secondary 42 B30.

Key words and phrases. Locally integrable structures, Baouendi-Treves approximation formula, Hardy spaces, CR functions.

This work was supported in part by CNPq and FAPESP. 
(1) $\sup _{x \in X}\left\|F_{j}(x+i \cdot)\right\|_{h^{p}} \leq C\|F\|_{L^{\infty}\left(X, h^{p}\left(\mathbb{R}^{m}\right)\right)}$,
(2) $\lim _{j \rightarrow \infty}\left\|F_{j}(x+i \cdot)-F(x+i \cdot)\right\|_{h^{p}}=0, x \in X$.

When $X \subset \mathbb{R}^{m}$ is an embedded submanifold it is standard to define CR functions as solutions of the tangential Cauchy-Riemann equations (on the subject of CR manifolds we refer to B1]). That both definitions agree in the regular case, when $X$ is a connected submanifold, follows from an appropriate version of the BaouendiTreves approximation theorem [BT] (see Proposition A.1 in the appendix). One of the consequences of the extension results we prove may be stated as follows (the interior is taken with respect to the affine subspace generated by $\operatorname{ch}(X))$ :

Theorem A. Assume that $X=\Phi(N) \subset \mathbb{R}^{m}$ is the image of a $C^{1}$ map $\Phi$ : $N \longrightarrow \mathbb{R}^{m}$ that takes a connected $C^{1}$ manifold $N$ into $\mathbb{R}^{m}$ and $0<p<\infty$. Then any generalized $C R$ function $g \in L^{\infty}\left(X, h^{p}\left(\mathbb{R}^{m}\right)\right.$ ) has a generalized $C R$ extension $g^{b} \in L^{\infty}\left(\operatorname{ch}(X), h^{p}\left(\mathbb{R}^{m}\right)\right)$. Furthermore, the map

$$
\operatorname{Int} \operatorname{ch}(X) \ni x \longmapsto g^{b}(x) \in h^{p}\left(\mathbb{R}^{m}\right)
$$

is continuous and satisfies

$$
\left\|g^{b}\right\|_{L^{\infty}\left(\operatorname{ch}(X), h^{p}\left(\mathbb{R}^{m}\right)\right)}=\|g\|_{L^{\infty}\left(X, h^{p}\left(\mathbb{R}^{m}\right)\right)} .
$$

If the affine subspace generated by $\operatorname{ch}(X)$ has dimension $m$, then $g^{b}$ is holomorphic on the open tube $\operatorname{Int} \operatorname{ch}(X)+i \mathbb{R}^{m}$.

In particular, $X$ is allowed to be a polyhedron, a self-intersecting polygonal line or, more generally, a submanifold with intersections, corners and cusps.

The extension $g^{b}$ obtained in Theorem A has the following regularity property. For any pair of distinct points $x_{1}, x_{2} \in X$, consider the one-dimensional strip

$$
S\left(x_{1}, x_{2}\right)=\left\{x_{1}+(s+i t)\left(x_{2}-x_{1}\right), \quad 0<s<1, t \in \mathbb{R}\right\} \subset \mathbb{C}^{m} .
$$

Then $g^{b}$ may be regarded as an $\mathcal{S}^{\prime}\left(\mathbb{R}^{m-1}\right)$-valued holomorphic function defined on $S\left(x_{1}, x_{2}\right)$ whose boundary values on the sides of the strip are determined by $g$. It follows that $g^{b}$ is the only extension of $g$ with that regularity property.

Although $g^{b}$ might not be continuous at points $x_{0} \in X \backslash \operatorname{Int} \operatorname{ch}(X)$, the function $g$ may be recovered from the restriction of $g^{b}$ to $\operatorname{Int} \operatorname{ch}(X)$ by letting $x_{0}$ be approached "nontangentially" by points in $\operatorname{Int} \operatorname{ch}(X)$. Here we use a much weaker notion of nontangential approach than the usual one (see Definition 3.1 and Theorem 3.1 for precise definitions and statements).

The paper is organized as follows. In section 1 we briefly discuss locally integrable tube structures while the appropriate versions of the Baouendi-Treves approximation formula that will be needed in the context of Hardy spaces on tubes are discussed in the appendix. Section 2 is the technical core of the paper where we define generalized $\mathrm{CR}$ functions on rough tubes and study two-point convergence in $L^{p}$ and $h^{p}$ by subaveraging techniques related to those used in [B2]. Section 3 is devoted to the statement and proof of several extension results that, in particular, imply Theorem A above. Finally, in section 4 we show that the extension function produced in section 4 has a regularity property that singles it out among all other extensions. 


\section{Locally integrable tube structures}

Let $M$ be a manifold of class $C^{k}, 1 \leq k \leq \infty$, and dimension $\nu$. A complex vector subbundle $\mathcal{V}$ of $\mathbb{C} T M$, of rank $n$, is said to define a locally integrable structure of class $C^{k}$ if given an arbitrary point $p \in M$ there exist an open neighborhood $U$ of $p$ and functions $Z_{1}, \ldots, Z_{m} \in C^{k}(U), m=\nu-n$, with linearly independent differentials such that

$$
\operatorname{span}\left\{d Z_{1}(p), \ldots, d Z_{m}(p)\right\}=\mathcal{V}_{p}^{\perp}, \quad p \in U .
$$

The functions $Z_{1}, \ldots, Z_{m}$ are called local first integrals of the structure and the number $m$ is called the co-rank of $\mathcal{V}$. It is known (see, e.g., [BCH, Corollary I.10.2] for the case $k=\infty)$ that we can always find local coordinates $\left(x_{1}, \ldots, x_{m}, t_{1}, \ldots, t_{n}\right)$ vanishing at $p$ in terms of which $Z_{1}, \ldots, Z_{m}$ have the form

$$
Z_{k}(x, t)=x_{k}+i \phi_{k}(x, t), \quad k=1, \ldots, m,
$$

and the functions $\phi_{k}, k=1, \ldots, m$ are real-valued and of class $C^{k}$ and satisfy

$$
\phi_{k}(0,0)=0, \quad d_{x} \phi_{k}(0,0)=0, \quad k=1, \ldots, m .
$$

A manifold $M$ of the form $M=N \times \mathbb{R}^{m}$, where $N$ is a manifold of dimension $n$ and class $C^{k}$ will be called a tube manifold. A locally integrable structure $\mathcal{V}$ on $\mathbb{C} T M$ is called a tube structure on $M$ if for any point $q \in N$ there exist local coordinates $t_{1}, \ldots, t_{n}$ in a neighborhood $V$ of $q$ in $N$ and real-valued functions $\phi_{k}(t), k=1, \ldots, m$, of class $C^{k}$ defined on $V$, satisfying $\phi_{k}(0)=0$, such that the differentials $d Z_{k}$ of the functions

$$
Z_{k}(x, t)=x_{k}+i \phi_{k}(t), \quad k=1, \ldots, m
$$

span $\mathcal{V}^{\perp}$ on the tube over $V$, i.e., on the set $V \times \mathbb{R}^{m}$. It follows that $\mathcal{V}$ is spanned on the tube over $V$ by the $n$ vector fields of class $C^{k-1}$ :

$$
\begin{aligned}
L_{j} & =\frac{\partial}{\partial t_{j}}+\sum_{k=1}^{m} \lambda_{j k}(t) \frac{\partial}{\partial x_{k}}, \quad j=1, \ldots, n, \\
\lambda_{j k}(t) & =-i \frac{\partial \phi_{k}}{\partial t_{j}}(t), \quad j=1, \ldots, n, \quad k=1, \ldots, m .
\end{aligned}
$$

The main feature about the vector fields (1.2) is that the coefficients are independent of $x$. Hence, in spite of the fact that they might be continuous and not differentiable (when $k=1$ ), it is possible to apply them to distributions which are arbitrarily irregular with respect to the variable $x$. We denote by $L^{\infty}\left(N, \mathcal{S}^{\prime}\left(\mathbb{R}^{m}\right)\right)$ the space of bounded $\mathcal{S}^{\prime}\left(\mathbb{R}^{m}\right)$-valued weakly measurable functions defined on $N$. More precisely, if $t$ denotes a generic point in $N$ and $x$ a generic point in $\mathbb{R}^{m}, f(t, x)$ belongs to $L^{\infty}\left(N, \mathcal{S}^{\prime}\left(\mathbb{R}^{m}\right)\right)$ if and only if

(1) for every $\psi(x) \in C_{c}^{\infty}\left(\mathbb{R}^{m}\right)$, the map

$$
N \ni t \mapsto\langle f(t, \cdot), \psi(\cdot)\rangle
$$

is measurable.

(2) There exists a negligible set $N_{0} \subset N$ such that

$$
\bigcup_{t \in N \backslash N_{0}} f(t, \cdot)
$$

is a weakly bounded set of $\mathcal{S}^{\prime}\left(\mathbb{R}^{m}\right)$. 
Remark 1.1. Later we will show that when $f \in L^{\infty}\left(N, \mathcal{S}^{\prime}\left(\mathbb{R}^{m}\right)\right)$ is a homogeneous solution of the structure we may always take the set $N_{0}$ as the empty set.

In the next section we will describe the Baouendi-Treves approximation formula for distributions $f(x, t) \in L^{\infty}\left(N, \mathcal{S}^{\prime}\left(\mathbb{R}^{m}\right)\right)$ that are homogeneous solutions of the tube structure $\mathcal{V}$. On local coordinates $(x, t)$, where $\mathcal{V}$ is generated by the vector fields (1.2), this means that

$$
\int\left\langle f(\cdot, t), L_{j} \psi(\cdot, t)\right\rangle d t=0, \quad \psi(x, t) \in C_{c}^{\infty}\left(V \times \mathbb{R}^{m}\right), \quad j=1, \ldots, n,
$$

where $\langle$,$\rangle denotes the duality pairing between tempered distributions and test$ functions in $\mathbb{R}^{m}$.

\section{THE EXTENSION PROBLEM}

From now on, we will always assume that the manifold $N$ is connected. We know from Proposition A.1 in the appendix that to every homogeneous solution $f \in L^{\infty}\left(N, h^{p}\left(\mathbb{R}^{m}\right)\right)$ of a globally integrable tube structure there corresponds a unique function $\tilde{f} \in L^{\infty}\left(\tilde{N}, h^{p}\left(\mathbb{R}^{m}\right)\right)$ such that $f=\tilde{f} \circ(i Z)$. Since $\tilde{f}$ is the limit of restrictions to $\tilde{M} \doteq \tilde{N}+i \mathbb{R}^{m}$ of entire functions, we may regard $\tilde{f}$ as a generalized CR distribution on the generalized tube $\tilde{M}$, in spite of the fact that $\tilde{M}$ may not be a submanifold: all we know about $\tilde{N}$ is that it is the image of a manifold $N$ of dimension $n$ under a differentiable map into $\mathbb{R}^{m}$ with $n$ smaller than, equal to, or larger than $m$. For instance, in the important case of CR tube submanifolds mentioned in Example A.1, $\tilde{N}$ is an embedded submanifold of $\mathbb{R}^{m}$, in Example A.2, $\tilde{N}=(-\infty, 0]$ is a manifold with boundary that may be regarded as a folded copy of $\mathbb{R}$, and in Example A.3, $\tilde{N}$ has empty interior and is not closed. In general, $\tilde{N}$ is allowed to have self-intersections and cusps.

Definition 2.1. Let $X \subset \mathbb{R}^{m}$ be a measurable set. Given $F \in L^{\infty}\left(X, h^{p}\left(\mathbb{R}^{m}\right)\right)$, regard it as a distribution $F(x+i y)$ defined on the tube $X+i \mathbb{R}^{m}$. We say that $F$ is a (generalized) $\mathrm{CR}$ function if there exists a sequence $F_{j}(x+i y)$ of entire functions in $\mathbb{C}^{m}$ such that

(1) $\sup _{x \in X}\left\|F_{j}(x+i \cdot)\right\|_{h^{p}} \leq C\|F\|_{L^{\infty}\left(X, h^{p}\left(\mathbb{R}^{m}\right)\right)}$,

(2) $\lim _{j \rightarrow \infty}\left\|F_{j}(x+i \cdot)-F(x+i \cdot)\right\|_{h^{p}}=0, x \in X$.

Note that when $X \subset \mathbb{R}^{m}$ is a connected immersed submanifold, the tangential Cauchy-Riemann equations are well defined on $X+i \mathbb{R}^{m}$ and we have a standard notion of CR function on $X+i \mathbb{R}^{m}$. In this case, the classical definition coincides with the one given by Definition 2.1 by an application of Proposition A.1. Loosely speaking, the extension problem for $F$ is that of extending $F$ to a larger set $X^{\prime} \supset$ $X$ preserving its generalized $\mathrm{CR}$ character. A natural approach is to determine whether the sequence of entire functions $F_{j}$, which converges in an appropriate way to $F$ on $X$, also converges on a larger set $X^{\prime}$. We start by studying two-point convergence in $L^{p}$ norms.

2.1. Convergence in $L^{p}$. Our main focus will be the case $0<p \leq 1$. Consider a sequence of entire functions $f_{j}(z), z \in \mathbb{C}^{m}, m \geq 2$. Let us write $g_{j}(y)=f_{j}(0+i y)$, $h_{j}(y)=f_{j}(e+i y), y \in \mathbb{R}^{m}$, where $e=\left(a_{1}, \ldots, a_{m}\right) \in \mathbb{R}^{m}$ is a fixed vector. Our 
main hypothesis will be that, for some fixed $0<p \leq 1$, and some continuous functions $c_{j}(x)>0, x \in \mathbb{R}^{m}, j=1,2, \ldots$,

$$
\begin{aligned}
& \left|f_{j}(x+i y)\right| \leq c_{j}(x), \quad x, y \in \mathbb{R}^{m}, \quad j=1,2, \ldots, \\
& \lim _{j, k \rightarrow \infty} \int_{\mathbb{R}^{m}}\left|g_{j}-g_{k}\right|^{p}+\left|h_{j}-h_{k}\right|^{p} d y=0 .
\end{aligned}
$$

Note that (2.2) means that for $x$ fixed the sequences $\left(g_{j}\right)$ and $\left(h_{j}\right)$ are Cauchy sequences for the $p$-gauge as functions of $y$. Consider the holomorphic function of one variable $w \in \Delta=\{|w|<1\}$, depending on a real parameter $0 \leq t \leq 1$ given by

$$
K(w, t)=\frac{1}{2 \pi} \int_{0}^{2 \pi t} \frac{e^{i \theta}+w}{e^{i \theta}-w} d \theta, \quad|w|<1, \quad 0<t<1 .
$$

Note that $K(0, t)=t, K(w, 0) \equiv 0$ and $K(w, 1) \equiv 1$. Moreover, $K(w, t) \in H^{2}(\Delta)$ for fixed $t \in(0,1)$ and its boundary value $b K(w, t)$ as a function of $w \in \Delta$ is

$$
b K\left(e^{i \theta}, t\right)=\chi(\theta, t)+i(H \chi)\left(e^{i \theta}, t\right),
$$

where $[0,2 \pi] \ni \theta \mapsto \chi(\theta, t)$ is the characteristic function of the interval $(0,2 \pi t)$ and $H \chi$ its Hilbert transform (on the subject of Hardy spaces $H^{p}(\Delta)$ on the unit disc we refer to $[\mathrm{Du}])$. It follows that $\Re b K\left(e^{i \theta}, t\right)$ is 1 if $0<\theta<2 \pi t$ and is 0 if $2 \pi t \leq \theta \leq 2 \pi$, so $\Re b K\left(S^{1}, t\right) \subset\{0,1\}, 0<t<1$. Furthermore, $b K\left(e^{i \theta}, t\right)$ is continuous for $\theta \neq 0, t$, so $b K\left(e^{i \theta}, t\right)=\lim _{r \nearrow 1} b K\left(r e^{i \theta}, t\right)$ except at those exceptional values. To simplify the notation, from now on we will write $K\left(e^{i \theta}, t\right)$ rather than $b K\left(e^{i \theta}, t\right)$. For fixed $z=t e+i y \in \mathbb{C}^{m}, 0 \leq t \leq 1,\left(y_{1}, \ldots, y_{m}\right) \in \mathbb{R}^{m}$ and $e=\left(a_{1}, \ldots, a_{m}\right) \in \mathbb{R}^{m}$ set

$$
\begin{aligned}
U_{j}(w, z) & =f_{j}(K(w, t) e+i y) \\
& =f_{j}\left(K(w, t) a_{1}+i y_{1}, \ldots, K(w, t) a_{m}+i y_{m}\right),
\end{aligned}
$$

$|w|<1$. It follows that

$$
U_{j}(0, z)=f_{j}(K(0, t) e+i y)=f_{j}(z) .
$$

Lemma 2.1. For fixed $t \in(0,1),\left(y_{1}, \ldots, y_{m}\right) \in \mathbb{R}^{m}$, the function

$$
v_{j}(w)=U_{j}(w, t e+i y)
$$

is in the Hardy space $H^{p}(\Delta)$. In particular, for any $c \in \mathbb{C}$,

$$
\left|v_{j}(0)-c\right|^{p} \leq \frac{1}{2 \pi} \int_{0}^{2 \pi}\left|b v_{j}\left(e^{i \theta}\right)-c\right|^{p} d \theta .
$$

Proof. We will assume initially that $c=0$. The function $\Delta \ni w \mapsto U_{j}(w, x+i y)$ is holomorphic and $\left|v_{j}(w)\right|^{p}$ is subharmonic on $\Delta$. The set $\{K(w, t) e:|w|<1\}$ is contained in the set $\{|x| \leq|e|\}+i \mathbb{R}^{m}$, so (2.1) shows that $v_{j}(w)$ is bounded and belongs to $H^{\infty}(\Delta) \subset H^{p}(\Delta)$. In fact,

$$
\left|v_{j}(w)\right|=\left|f_{j}(K(w, t) e+i y)\right| \leq c_{j}(\Re K(w, t) e) .
$$

It is clear that

$$
\left|v_{j}(0)\right|^{p} \leq \frac{1}{2 \pi} \int_{0}^{2 \pi}\left|v_{j}\left(r e^{i \theta}\right)\right|^{p} d \theta, \quad 0<r<1
$$

because $\left|v_{j}(w)\right|^{p}$ is subharmonic and, letting $r \nearrow 1$, the right hand side converges to the right hand side of (2.3) (since $v_{j}(w)$ is bounded this is an easy instance of a well-known property of Hardy spaces [Du, p.21]). In the general case, we reason as before with the function $v_{j}(w)-c$. 
We next rewrite (2.3) as

$$
\left|U_{j}(0, t e+i y)\right|^{p} \leq \frac{1}{2 \pi} \int_{0}^{2 \pi}\left|U_{j}\left(e^{i \theta}, t e+i y\right)\right|^{p} d \theta
$$

or

$$
\left|f_{j}(t e+i y)\right|^{p} \leq \frac{1}{2 \pi} \int_{0}^{2 \pi}\left|f_{j}\left(K\left(e^{i \theta}, t\right) e+i y\right)\right|^{p} d \theta .
$$

We are going to integrate the above inequality with respect to $y \in \mathbb{R}^{m}$ and apply Fubini's theorem to the right hand side. In particular,

$$
\int_{\mathbb{R}^{m}}\left|f_{j}\left(K\left(e^{i \theta}, t\right) e+i y\right)\right|^{p} d y=\int_{\mathbb{R}^{m}}\left|f_{j}(\chi(\theta, t) e+i y)\right|^{p} d y .
$$

Now, $\chi(\theta, t)$ is either 1 or 0 depending on whether $\theta<2 t \pi$ or $\theta \geq 2 t \pi$. Thus,

$$
\begin{aligned}
\int_{\mathbb{R}^{m}} \mid f_{j}(\chi(\theta, t) e & +i y)\left.\right|^{p} d y \\
& \leq \int_{\mathbb{R}^{m}} \chi(\theta, t)\left|f_{j}(e+i y)\right|^{p} d y+\int_{\mathbb{R}^{m}}(1-\chi(\theta, t))\left|f_{j}(0+i y)\right|^{p} d y .
\end{aligned}
$$

Integrating this estimate with respect to $\theta \in S^{1}$ we get

$$
\begin{aligned}
\int_{\mathbb{R}^{m}}\left|f_{j}(t e+i y)\right|^{p} d y \leq t \int_{\mathbb{R}} \mid & \left.f_{j}(e+i y)\right|^{p} d y \\
& +(1-t) \int_{\mathbb{R}^{m}}\left|f_{j}(0+i y)\right|^{p} d y .
\end{aligned}
$$

Of course, the same argument could be applied to the difference $f_{j}-f_{k}$, which, taking account of (2.2), implies that for $t \in(0,1)$ fixed, $y \mapsto f_{j}\left(t e_{1}+i y\right)$ is a Cauchy sequence in $L^{p}\left(\mathbb{R}^{m}\right)$, something we already knew for the endpoints of the interval. Summing up, for any $t \in[0,1], f_{j}(t e+i y)$ converges in $L^{p}\left(\mathbb{R}^{m}\right)$ to a function $f(t e+i y)$ and

$$
\|f(t e+i \cdot)\|_{L^{p}}^{p} \leq t\|f(e+i \cdot)\|_{L^{p}}^{p}+(1-t)\|f(0+i \cdot)\|_{L^{p}}^{p},
$$

as follows from letting $j \rightarrow \infty$ in (2.5). Notice that an estimate analogous to (2.6) will hold if we replace the endpoints 0 and $e$ by any other pair of points belonging to the segment joining 0 to $e$ and this means that $t \mapsto\|f(t e+i \cdot)\|_{L^{p}}^{p}$ is a convex function of $t \in[0,1]$. This proves, in particular, that $t \mapsto\|f(t e+i \cdot)\|_{L^{p}}^{p}$ is continuous on the open interval $(0,1)$. The continuity at the endpoints needs more care and will be proved next. Applying (2.3) to

$$
v_{j}(w)=U_{j}(w, t e+i y), \quad y \in \mathbb{R}^{m}
$$

with $c=f(0+i y)$ we get, for $y \in \mathbb{R}^{m}$,

$$
\begin{aligned}
\mid f_{j}(t e+i y)- & \left.f(0+i y)\right|^{p} \\
\leq & \frac{1}{2 \pi} \int_{0}^{2 \pi}\left|f_{j}\left(K\left(e^{i \theta}, t\right) e+i y\right)-f(0+i y)\right|^{p} d \theta .
\end{aligned}
$$


Integrating this estimate with respect to $y$, reasoning as we did to prove (2.5) and applying Fubini's theorem, we obtain

$$
\begin{aligned}
& \left\|f_{j}(t e+i \cdot)-f(0+i \cdot)\right\|_{L^{p}}^{p} \\
& \quad \leq \frac{1}{2 \pi} \int_{0}^{2 \pi} \int_{\mathbb{R}^{m}}\left|f_{j}\left(K\left(e^{i \theta}, t\right) e+i y\right)-f(0+i y)\right|^{p} d y d \theta .
\end{aligned}
$$

To estimate the integral

$$
\int_{\mathbb{R}^{m}}\left|f_{j}\left(K\left(e^{i \theta}, t\right) e+i y\right)-f(0+i y)\right|^{p} d y
$$

we proceed as follows. Adding and subtracting the term

$$
f\left(K\left(e^{i \theta}, t\right) e+i y\right)
$$

inside the integral (2.8) and using the subadditivity of the function $\mathbb{R}^{+} \ni t \mapsto t^{p}$ for $0<p \leq 1$, we obtain

$$
\begin{aligned}
& \int_{\mathbb{R}^{m}} \mid f_{j}\left(K\left(e^{i \theta}, t\right) e+i y\right)-\left.f(0+i y)\right|^{p} d y \\
& \leq \int_{\mathbb{R}^{m}}\left|f_{j}\left(K\left(e^{i \theta}, t\right) e+i y\right)-f\left(K\left(e^{i \theta}, t\right) e+i y\right)\right|^{p} d y \\
& \quad+\int_{\mathbb{R}^{m}}\left|f\left(K\left(e^{i \theta}, t\right) e+i y\right)-f(0+i y)\right|^{p} d y .
\end{aligned}
$$

Thus, we may write

$$
\begin{aligned}
& \int_{\mathbb{R}^{m}} \mid f_{j}\left(K\left(e^{i \theta}, t\right) e+i y\right)-\left.f(0+i y)\right|^{p} d y \\
& \leq \int_{\mathbb{R}^{m}}\left|f_{j}(\chi(\theta, t) e+i y)-f(\chi(\theta, t) e+i y)\right|^{p} d y+g_{e}(\theta, t),
\end{aligned}
$$

where

$$
g_{e}(\theta, t) \doteq \int_{\mathbb{R}^{m}}\left|f\left(K\left(e^{i \theta}, t\right) e+i y\right)-f(0+i y)\right|^{p} d y .
$$

Integrating the last estimate with respect to $\theta$ and taking account of (2.7) we obtain

$$
\begin{gathered}
\left\|f_{j}(t e+i \cdot)-f(0+i \cdot)\right\|_{L^{p}}^{p} \\
\leq \frac{1}{2 \pi} \int_{0}^{2 \pi} \int_{\mathbb{R}^{m}}\left|f_{j}(\chi(\theta, t) e+i y)-f(\chi(\theta, t) e+i y)\right|^{p} d y d \theta \\
\quad+\frac{1}{2 \pi} \int_{0}^{2 \pi} g_{e}(\theta, t) d \theta \\
=t\left\|\left(f_{j}-f\right)(e+i \cdot)\right\|_{L^{p}}^{p}+(1-t)\left\|\left(f_{j}-f\right)(0+i \cdot)\right\|_{L^{p}}^{p} \\
\quad+\frac{1}{2 \pi} \int_{0}^{2 \pi} g_{e}(\theta, t) d \theta .
\end{gathered}
$$

Letting $j \rightarrow \infty$ we derive

$$
\|f(t e+i \cdot)-f(0+i \cdot)\|_{L^{p}}^{p} \leq \frac{1}{2 \pi} \int_{0}^{2 \pi} g_{e}(\theta, t) d \theta .
$$

Note that

$$
g_{e}(\theta, t) \leq \int_{\mathbb{R}^{m}}|f(\chi(\theta, t) e+i y)|^{p} d y+\|f(0+i \cdot)\|_{L^{p}}^{p} .
$$


Thus,

$$
g_{e}(\theta, t) \leq\|f(e+i \cdot)\|_{L^{p}}^{p}+2\|f(0+i \cdot)\|_{L^{p}}^{p} \leq C
$$

for each $\theta \in[0,2 \pi]$ and $0 \leq t \leq 1$. We will now show that $g(\theta, t) \rightarrow 0$ as $t \searrow 0$ for $0<\theta<2 \pi$. Indeed, since $y \mapsto f(0+i y) \in L^{p}\left(\mathbb{R}^{n}\right)$, we know that

$$
\lim _{h \rightarrow 0} \int_{\mathbb{R}^{m}}|f(0+i(y+h))-f(0+i y)|^{p} d y=0 .
$$

On the other hand, for $0<\theta<2 \pi$ and $0<t<\theta, K\left(e^{i \theta}, t\right)=i(H \chi)\left(e^{i \theta}, t\right)$ and then the standard integral formula for $(H \chi)\left(e^{i \theta}, t\right)$ allows us to conclude that $\lim _{t \searrow 0} K\left(e^{i \theta}, t\right)=0$ if $0<\theta<2 \pi$. In particular, $g_{e}(\theta, t) \rightarrow 0$ as $t \searrow 0$ for $0<\theta<2 \pi$. In view of (2.11), the dominated convergence theorem shows that

$$
\lim _{t \searrow 0} \frac{1}{2 \pi} \int_{0}^{2 \pi} g_{e}(\theta, t) d \theta=0 .
$$

Finally, (2.10) tells us that

$$
\lim _{t \searrow 0}\|f(t e+i \cdot)-f(0+i \cdot)\|_{L^{p}}^{p}=0 .
$$

Of course, a similar argument can be applied to the other endpoint to show that

$$
\lim _{t \nearrow 1}\|f(t e+i \cdot)-f(e+i \cdot)\|_{L^{p}}^{p}=0 .
$$

Thus, $t \mapsto\|f(t e+i \cdot)\|_{L^{p}}^{p}$ is continuous on the closed interval $[0,1]$.

Summing up, we have proved that if $x_{1}, x_{2} \in \mathbb{R}^{m}$ are such that $y \mapsto f_{j}\left(x_{k}+i y\right)$ converges in $L^{p}\left(\mathbb{R}^{m}\right), k=1,2$, then $f_{j}(x+i y)$ also converges in $L^{p}\left(\mathbb{R}^{m}\right)$ for $x \in$ $\left(x_{1}, x_{2}\right)$, the open segment joining $x_{1}$ and $x_{2}$, and the limit yields a continuous function on $\left[x_{1}, x_{2}\right]$ with values in $L^{p}\left(\mathbb{R}^{m}\right)$.

2.2. Convergence in $h^{p}$. The arguments of the previous subsection can be applied with small changes to the case of $h^{p}$ "norms" (see Definition A.1 in the appendix) as we briefly describe below. Once again, we direct our attention to the case $0<p \leq 1$, although the conclusions remain true and are easier to prove in the case of Lebesgue spaces $p>1$. Suppose that (2.1) holds but instead of (2.2) we have the stronger estimate

$$
\lim _{j, k \rightarrow \infty} \int_{\mathbb{R}^{m}} m_{\varphi}\left(g_{j}-g_{k}\right)^{p}+m_{\varphi}\left(h_{j}-h_{k}\right)^{p} d y=0 ;
$$

i.e., $\left(g_{k}\right)$ and $\left(h_{k}\right)$ are Cauchy sequences in $h^{p}\left(\mathbb{R}^{m}\right)$. Then, we may apply the $L^{p}$ estimate (2.4) to the entire functions

$$
\tilde{f}_{j}(x+i y) \doteq\left(f_{j} * \varphi_{\varepsilon}\right)(y)=\int_{\mathbb{R}^{m}} f_{j}\left(x+i\left(y-y^{\prime}\right)\right) \varphi_{\varepsilon}\left(y^{\prime}\right) d y^{\prime}
$$

that satisfy (2.1) and (2.2) to obtain

$$
\left|\left(f_{j} * \varphi_{\varepsilon}\right)(t e+i y)\right|^{p} \leq \frac{1}{2 \pi} \int_{0}^{2 \pi}\left|\left(f_{j}^{(y)} \varphi_{\varepsilon}\right)\left(K\left(e^{i \theta}, t\right) e+i y\right)\right|^{p} d \theta .
$$

Now we take the sup in $0<\epsilon<1$ in the above inequality to get

$$
\left|m_{\varphi} f_{j}(t e+i y)\right|^{p} \leq \frac{1}{2 \pi} \int_{0}^{2 \pi}\left|m_{\varphi} f_{j}\left(K\left(e^{i \theta}, t\right) e+i y\right)\right|^{p} d \theta .
$$


Now we integrate the last estimate with respect to $y \in \mathbb{R}^{m}$, to wit

$$
\begin{aligned}
\int_{\mathbb{R}^{m}}\left|m_{\varphi} f_{j}(t e+i y)\right|^{p} d y \leq t & \int_{\mathbb{R}^{m}}\left(m_{\varphi} f_{j}\right)^{p}(e+i y) \\
& +(1-t) \int_{\mathbb{R}^{m}}\left(m_{\varphi} f_{j}\right)^{p}(0+i y) d y .
\end{aligned}
$$

This proves that

$$
\|f(t e+i \cdot)\|_{h^{p}}^{p} \leq t\|f(t e+i \cdot)\|_{h^{p}}^{p}+(1-t)\|f(0+i \cdot)\|_{h^{p}}^{p} .
$$

Similarly, we may prove that

$$
\begin{aligned}
& \lim _{t \searrow 0}\|f(t e+i \cdot)-f(0+i \cdot)\|_{h^{p}}^{p}=0, \\
& \lim _{t \nearrow 1}\|f(t e+i \cdot)-f(e+i \cdot)\|_{h^{p}}^{p}=0 .
\end{aligned}
$$

\section{Extension theOREMS}

We start by considering a general notion of nontangential approach that is weaker than the standard notion of nontangential approach for boundary points of smoothly bounded open subsets of $\mathbb{R}^{m}$.

Definition 3.1. Let $S \subset \mathbb{R}^{m}$ be a set. We will say that a sequence $\left(x_{j}\right)$ in $S$ approaches $x_{0} \in S$ nontangentially in $S$ if there exist a sequence of numbers $\left(t_{j}\right)$, $0 \leq t_{j} \leq 1$ and a bounded sequence of vectors $\left(e_{j}\right)$ in $\mathbb{R}^{m}$ such that $x_{j}-x_{0}=t_{j} e_{j}$, $x_{0}+e_{j} \in S$ and $t_{j} \rightarrow 0$.

Note that if $x_{0}$ is an interior point of $S$, any sequence $\left(x_{j}\right)$ in $S$ that converges to $x_{0}$ will approach $x_{0}$ nontangentially in $S$, so the definition is only relevant for boundary points $x_{0} \in \partial S$. If $\Omega \subset \mathbb{R}^{m}$ is a smoothly bounded open set, $x_{0} \in \partial \Omega$, and $\left(x_{j}\right)$ is a sequence in $\Omega$ that approaches $x_{0}$ nontangentially in the standard sense, that is, there is an acute truncated convex cone $\Gamma \subset \Omega$ with vertex at $x_{0}$ such that $x_{j} \in \Gamma, j \in \mathbb{N}$, then $\left(x_{j}\right)$ approaches $x_{0}$ nontangentially in the sense of Definition 3.1. The converse is false, in general, so the notion on nontangential approach given by Definition 3.1 is less restrictive than the usual one.

Example 3.1. If $\Pi \subset \mathbb{R}^{m}$ is a closed half space,

$$
\left\{x \in \mathbb{R}^{m}: \quad x \cdot a \leq x_{0} \cdot a\right\},
$$

then any sequence $\left(x_{j}\right)$ in $\Pi$ that converges to $x_{0}$ approaches $x_{0}$ nontangentially in $\Pi$. More generally, any sequence $\left(x_{j}\right)$ in a closed polyhedron $P$ (i.e., $P$ is a finite, nonempty intersection of closed half spaces) that converges to a boundary point $x_{0} \in \partial P$ approaches $x_{0}$ nontangentially in $P$.

Example 3.2. If

$$
S=\left\{\left(x_{1}, x_{2}\right) \in \mathbb{R}^{2}: \quad x_{2} \geq x_{1}^{2}\right\},
$$

then the sequence $\left(x_{j}\right), x_{j}=\left(1 / j, 1 / j^{2}\right)$, converges to $x_{0}=(0,0) \in S$ but it does not approach $x_{0}$ nontangentially in $S$. On the other hand, the sequence $y_{j}=\left(1 / j, 1 / j^{3 / 2}\right)$ approaches $x_{0}$ nontangentially in $S$ (although it does not in the classical sense). 
Consider a sequence $\left(f_{j}(x+i y)\right)$ of entire functions in $\mathbb{C}^{m}$ satisfying (2.1) and suppose that $y \mapsto f_{j}(x+i y)$ converges in $h^{p}\left(\mathbb{R}^{m}\right)$ whenever $x \in X$, where $X$ is some measurable subset of $\mathbb{R}^{m}$. Also assume that

$$
\sup _{x \in X} \sup _{j}\left\|f_{j}(x+i \cdot)\right\|_{h^{p}}^{p}<\infty .
$$

By passing to the limit we obtain a function $f(x) \in L^{\infty}\left(X, h^{p}\left(\mathbb{R}^{m}\right)\right)$. Let us denote by $\operatorname{ch}(X)$ the convex hull of $X$, i.e., the set of convex combinations $\sum_{k=1}^{N} t_{j} x_{j}$, $x_{j} \in X, t_{j} \geq 0, \sum_{k=1}^{N} t_{j}=1, N=1,2, \ldots$ The convex hull can also be obtained by considering first the set $X_{1} \subset \operatorname{ch}(X)$ of convex combinations with two terms $t x_{1}+(1-t) x_{2}, x_{1}, x_{2} \in X, 0 \leq t \leq 1$, then the set $X_{2} \subset \operatorname{ch}(X)$ of points $t x_{1}+(1-t) x_{2}, x_{1}, x_{2} \in X_{1}, 0 \leq t \leq 1$, and so on. By Carathéodory's theorem $[\mathrm{H}$, p.41], $X_{m}=\operatorname{ch}(X)$. Then, by the results in Section 2 the sequence converges in $h^{p}\left(\mathbb{R}^{m}\right)$ for $x \in \operatorname{ch}(X)$ yielding a function $f^{b}(x) \in L^{\infty}\left(\operatorname{ch}(X), h^{p}\left(\mathbb{R}^{m}\right)\right.$ ) (that may also be denoted by $\left.x \mapsto f^{b}(x+i \cdot), x \in \operatorname{ch}(X)\right)$ and extends $f(x)$. Furthermore, a closer look at the arguments that give the continuity of $f^{b}$ at the endpoints of an interval $\left[x_{1}, x_{2}\right], x_{1}, x_{2} \in X$, reveals that $f^{b}(x) \rightarrow f^{b}\left(x_{0}\right)$ as $x \rightarrow x_{0} \in \operatorname{ch}(X)$ if we restrict the way in which $x \in \operatorname{ch}(X)$ approaches $x_{0}$. We will prove that $f^{b}(x)$ is "nontangentially continuous" in the sense that $f^{b}(x) \rightarrow f^{b}\left(x_{0}\right)$ in $h^{p}\left(\mathbb{R}^{m}\right)$ if $x \rightarrow x_{0}$ nontangentially in $\operatorname{ch}(X)$. To see this, fix a point in $\operatorname{ch}(X)$, that without loss of generality we take to be the origin, and let us prove the nontangential continuity of $f^{b}(x)$ at $x=0$. We must show that if $\left(x_{j}\right)$ is a sequence and $x_{j} \rightarrow 0$ nontangentially in $\operatorname{ch}(X)$, then $\left\|f^{b}\left(x_{j}+i \cdot\right)-f^{b}(0+i \cdot)\right\|_{h^{p}}^{p} \rightarrow 0$. We may write $x_{j}=t_{j} e_{j}$ with $e_{j} \in \operatorname{ch}(X) \subset \mathbb{R}^{m}, 0 \leq t_{j} \leq 1,\left|e_{j}\right| \leq C$ and $t_{j} \rightarrow 0$ as $j \rightarrow \infty$. From now on, we simply write $f$ to denote both the original distribution $f$ and its extension $f^{b}$. With the notation of subsection 2.2, we must prove that $\left\|m_{\varphi}\left[f\left(x_{j}+i \cdot\right)-f(0+i \cdot)\right]\right\|_{L^{p}}^{p} \rightarrow 0$. By an estimate similar to (2.10) we have

$$
\left\|f\left(t_{j} e_{j}+i \cdot\right)-f(0+i \cdot)\right\|_{h^{p}}^{p} \leq \frac{1}{2 \pi} \int_{0}^{2 \pi} g_{j}\left(\theta, t_{j}\right) d \theta
$$

with

$$
g_{j}\left(\theta, t_{j}\right) \doteq \int_{\mathbb{R}^{m}}\left(m_{\varphi}\left[f\left(K\left(e^{i \theta}, t_{j}\right) e_{j}+i y\right)-f(0+i y)\right]\right)^{p} d y
$$

We have

$$
g_{j}\left(\theta, t_{j}\right) \leq\left\|f\left(e_{j}+i \cdot\right)\right\|_{h^{p}}^{p}+2\|f(0+i \cdot)\|_{L^{p}}^{p} \leq C .
$$

To see that $g_{j}\left(\theta, t_{j}\right) \rightarrow 0$ for a.e. $\theta \in[0,2 \pi]$, fix $0<\theta<2 \pi$ and take $j_{0}$ large enough so $t_{j}<\theta$ for $j \geq j_{0}$. Hence, for $j \geq j_{0}, K\left(e^{i \theta}, t_{j}\right)=i H \chi\left(e^{i \theta}, t_{j}\right)$ and we may write

$$
\begin{aligned}
g_{j}\left(\theta, t_{j}\right) & =\int_{\mathbb{R}^{m}}\left(m_{\varphi}\left[f\left(0+i\left(y+H \chi\left(e^{i \theta}, t_{j}\right) e_{j}\right)\right)-f(0+i y)\right]\right)^{p} d y \\
& =\| f\left(0+i\left(\cdot+\tau_{j}\right)-f(0+i \cdot) \|_{h^{p}}^{p}\right.
\end{aligned}
$$

with $\tau_{j}=H \chi\left(e^{i \theta}, t_{j}\right) e_{j}$. Since $\tau_{j} \rightarrow 0$ as $t_{j} \rightarrow 0$, and the distribution $y \mapsto f(0+i y)$ belongs to $h^{p}\left(\mathbb{R}^{m}\right)$, we see that $g_{j}\left(\theta, t_{j}\right) \rightarrow 0$, so the dominated convergence theorem and (3.2) show that

$$
\left\|f\left(t_{j} e_{j}+i \cdot\right)-f(0+i \cdot)\right\|_{h^{p}}^{p} \rightarrow 0
$$


Summing up,

Theorem 3.1. Let $X \subset \mathbb{R}^{m}$ be a measurable set, $0<p<\infty$, and consider a sequence $\left(f_{j}(x+i y)\right)$ of entire functions in $\mathbb{C}^{m}$ that satisfy (2.1) and (3.1). Assume that the sequence $y \mapsto f_{j}(x+i y)$ converges in $h^{p}\left(\mathbb{R}^{m}\right)$ as $j \rightarrow \infty$ for every $x \in X$, and call $f(x) \in L^{\infty}\left(X, h^{p}\left(\mathbb{R}^{m}\right)\right)$ the limit. Then the sequence $y \mapsto$ $f_{j}(x+i y)$ converges in $h^{p}\left(\mathbb{R}^{m}\right)$ for all $x \in \operatorname{ch}(X)$ and the limit yields an $h^{p}\left(\mathbb{R}^{m}\right)$ valued function $f^{b}(x), x \in \operatorname{ch}(X)$, that is nontangentially continuous on $\operatorname{ch}(X)$. Furthermore,

$$
\left\|f^{b}\right\|_{L^{\infty}\left(\operatorname{ch}(X), h^{p}\left(\mathbb{R}^{m}\right)\right)}=\|f\|_{L^{\infty}\left(X, h^{p}\left(\mathbb{R}^{m}\right)\right)} .
$$

Let $\mathcal{V}$ be a globally integrable tube structure on $N \times \mathbb{R}^{m}$ of class $C^{k}, 1 \leq k \leq \infty$, $N$ connected, with global integral $Z(x, t)=x+i \Phi(t), x \in \mathbb{R}^{m}, t \in N$. Set $\tilde{N}=$ $-\Phi(N)$ and consider a function $f \in L^{\infty}\left(N, h^{p}\left(\mathbb{R}^{m}\right)\right)$ that is also a homogeneous solution of $\mathcal{V}$. Let $\tilde{f} \in L^{\infty}\left(\tilde{N}, h^{p}(\mathbb{R})\right)$ be the generalized CR function defined on the tube $\tilde{M}=\tilde{N}+i \mathbb{R}^{m}$ granted by Proposition A.1. Then the sequence $\left(F_{j}(x+i y)\right)$ that according to Proposition A.1 converges to $\tilde{f}$ satisfies the hypotheses of Theorem 3.1. We have

Theorem 3.2. Assume that $0<p<\infty$. Given a homogeneous solution $f \in$ $L^{\infty}\left(N, h^{p}\left(\mathbb{R}^{m}\right)\right)$ of the tube structure $\mathcal{V}$, the associated generalized CR function $\tilde{f} \in L^{\infty}\left(\tilde{N}, h^{p}(\mathbb{R})\right)$ has a generalized CR extension $\tilde{f}^{b} \in L^{\infty}\left(\operatorname{ch}(\tilde{N}), h^{p}\left(\mathbb{R}^{m}\right)\right)$ that is nontangentially continuous on $\operatorname{ch}(\tilde{N})$ and satisfies

$$
\left\|\tilde{f}^{b}\right\|_{L^{\infty}\left(\operatorname{ch}(\tilde{N}), h^{p}\left(\mathbb{R}^{m}\right)\right)}=\|\tilde{f}\|_{L^{\infty}\left(\tilde{N}, h^{p}\left(\mathbb{R}^{m}\right)\right)}=\|f\|_{L^{\infty}\left(N, h^{p}\left(\mathbb{R}^{m}\right)\right)} .
$$

Corollary 3.1. Let $X \subset \mathbb{R}^{m}$ be the image of some differentiable map; i.e., there exists a connected $C^{1}$ manifold $N$ and a $C^{1}$ map $\Phi: N \longrightarrow \mathbb{R}^{m}$ such that $\Phi(N)=$ $X$. Then any generalized $C R$ function $g \in L^{\infty}\left(X, h^{p}(\mathbb{R})\right)$ has a generalized $C R$ extension $g^{b} \in L^{\infty}\left(\operatorname{ch}(X), h^{p}\left(\mathbb{R}^{m}\right)\right)$ that is nontangentially continuous on $\operatorname{ch}(X)$ and satisfies

$$
\left\|g^{b}\right\|_{L^{\infty}\left(\operatorname{ch}(X), h^{p}\left(\mathbb{R}^{m}\right)\right)}=\|g\|_{L^{\infty}\left(\tilde{N}, h^{p}\left(\mathbb{R}^{m}\right)\right)} .
$$

Proof. Define a globally integrable tube structure $\mathcal{V}$ on $N \times \mathbb{R}^{m}$ by declaring that $Z(x, t)=x+i \Phi(t), x \in \mathbb{R}, t \in N$, is a global first integral. It is enough to show that Theorem 3.2 can be applied with $f=g \circ(i Z)$ and $\tilde{f}=g$. To see this, note that there is a sequence of entire functions $G_{j}(x+i y)$ such that

$$
\begin{aligned}
& \sup _{x \in X}\left\|G_{j}(x+i \cdot)\right\|_{h^{p}} \leq\|g\|_{L^{\infty}\left(X, h^{p}\left(\mathbb{R}^{m}\right)\right)}, \\
& \lim _{j \rightarrow \infty}\left\|G_{j}(x+i \cdot)-g(x+i \cdot)\right\|_{h^{p}}=0, x \in X,
\end{aligned}
$$

because $g$ is a generalized CR function. Then the differentiable functions $G_{j} \circ(i Z)$ are homogeneous solutions of $\mathcal{V}$ and converge to $f=g \circ(i Z)$ in the sense of distributions, showing that $f$ is a homogeneous solution of $\mathcal{V}$.

We recall that a subset $W$ of $\mathbb{R}^{m}$ is called an affine subspace of $\mathbb{R}^{m}$ if it is the translate $W=x_{0}+L$ of a linear subspace $L$ and the dimension of $W$ is by definition the dimension of $L$. Given a set $S \subset \mathbb{R}^{m}$, the convex hull $\operatorname{ch}(S)$ is contained in an affine subspace $W$ of minimal dimension. Let us denote (following [BD] by 
Int $\operatorname{ch}(S)$ the interior of $\operatorname{ch}(S)$ relative to the subspace $W$. The following results are trivial to prove but worth mentioning.

Proposition 3.1. Let $S$ be a subset of $\mathbb{R}^{m}$, let $x_{0} \in \operatorname{Int} \operatorname{ch}(S)$ and consider a sequence $\left(x_{j}\right)$ in $\operatorname{ch}(S)$ such that $x_{j} \rightarrow x_{0}$ as $j \rightarrow \infty$. Then $\left(x_{j}\right)$ approaches $x_{0}$ nontangentially in $\operatorname{ch}(S)$.

Corollary 3.2. The restriction to $\operatorname{Int} \operatorname{ch}(X)$ of the extension function $F^{b}(x) \in$ $L^{\infty}\left(\operatorname{ch}(X), h^{p}\left(\mathbb{R}^{m}\right)\right)$ given by Theorem 3.1 is continuous.

\section{UNIQUENESS}

Consider a sequence of entire functions $f_{j}(z), z \in \mathbb{C}^{m}$, such that for some fixed $0<p \leq 1$, and some continuous functions $c_{j}(x)>0, x \in \mathbb{R}^{m}, j=1,2, \ldots$,

$$
\left|f_{j}(x+i y)\right| \leq c_{j}(x), \quad x, y \in \mathbb{R}^{m} \quad j=1,2, \ldots
$$

Assume further that, for some points $a \neq b \in \mathbb{R}^{m}$, the functions $y \mapsto f_{j}(a+i y)$ and $y \mapsto f_{j}(b+i y)$, are in $h^{p}\left(\mathbb{R}^{m}\right), j \in \mathbb{N}$, and that these two sequences are Cauchy sequences in $h^{p}\left(\mathbb{R}^{m}\right)$. We have seen in subsection 2.2 that the sequence $y \mapsto f_{j}(x+i y)$ converges in $h^{p}\left(\mathbb{R}^{m}\right)$ for any $x \in[a, b] \subset \mathbb{R}^{m}$ to a function that we denote (with some abuse of notation) by $f(x+i y), x \in[a, b], y \in \mathbb{R}^{m}$. We wish to determine how much regularity is inherited by the limit $f(x+i y)$ from the holomorphic functions $f_{j}(z)$. Let $\ell$ be the complex line determined by $a$ and $b$ and let $\Pi \subset \mathbb{C}^{m}$ be the complex hyperplane orthogonal to $\ell$ that passes through $a$. Choose an orthogonal map $R$ in $\mathbb{R}^{m}$ such that $R(|b-a|, 0, \ldots, 0)=b-a$ and $a+R\left(\{0\} \times \mathbb{R}^{m-1}\right)=\Pi \cap \mathbb{R}^{m}$. Extend $R$ by linearity to a unitary map on $\mathbb{C}^{m}$. Let $\psi \in C_{c}^{\infty}\left(\mathbb{R}^{m-1}\right)$ and set for $w=s+i t \in \mathbb{C}, z^{\prime}=0+i y^{\prime} \in \mathbb{C}^{m-1}$,

$$
f_{j}^{\psi}(w)=\int_{\mathbb{R}^{m-1}} f_{j}\left(a+R\left(w, i y^{\prime}\right)\right) \psi\left(y^{\prime}\right) d y^{\prime} .
$$

We are going to show that $\left(f_{j}^{\psi}(w)\right)$ is a sequence of entire functions of one variable with the following property: for any $0 \leq s \leq r \doteq|b-a|$, the sequence $t \mapsto f_{j}^{\psi}(s+i t)$ converges in $\mathcal{S}^{\prime}(\mathbb{R})$ to a tempered distribution $\mathcal{S}(\mathbb{R}) \ni u(t) \mapsto\left\langle f^{\psi}(s+i \cdot), u(\cdot)\right\rangle$. Using a linear change of variables, there is no loss of generality in assuming that $a=0, b=e_{1}=(1,0, \ldots, 0)$ and $R=I d$ as we do from now on.

Lemma 4.1. For each $s \in[0,1]$, the sequence of entire functions of one variable $t \mapsto f_{j}^{\psi}(s+i t)$ converges in $\mathcal{S}^{\prime}(\mathbb{R})$ to a tempered distribution $\mathcal{S}(\mathbb{R}) \ni u(t) \mapsto$ $\left\langle f^{\psi}(s+i \cdot), u(\cdot)\right\rangle$.

Proof. Notice that for each $u \in \mathcal{S}\left(\mathbb{R}_{t}\right)$,

$$
\begin{aligned}
\left\langle f_{j}^{\psi}(s+i \cdot), u(\cdot)\right\rangle & =\int_{\mathbb{R}} \int_{\mathbb{R}^{m-1}} f_{j}\left(s+i t, i y^{\prime}\right) \psi\left(y^{\prime}\right) u(t) d y^{\prime} d t \\
& =\int_{\mathbb{R}^{m}} f_{j}\left(s e_{1}+i y\right) \Phi(y) d y,
\end{aligned}
$$

where $\Phi(y)=(u \otimes \psi)\left(y_{1}, y^{\prime}\right)=u\left(y_{1}\right) \psi\left(y^{\prime}\right)$.

Since $y \mapsto f_{j}(x+i y)$ converges in $h^{p}\left(\mathbb{R}^{m}\right)$ for any $x \in[0,1] \subset \mathbb{R}^{m}$ to a function that we denote by $f(x+i y), x \in[a, b], y \in \mathbb{R}^{m}$, we see that

$$
\int_{\mathbb{R}^{m}} f_{j}\left(s e_{1}+i y\right) \Phi(y) d y \rightarrow\left\langle f\left(s e_{1}+i(\cdot)\right), \Phi(\cdot)\right\rangle
$$


as $j \rightarrow \infty$, so

$$
\left\langle f_{j}^{\psi}(s+i \cdot), u(\cdot)\right\rangle \rightarrow\left\langle f\left(s e_{1}+i(\cdot)\right), \Phi(\cdot)\right\rangle=\left\langle f\left(s e_{1}+i(\cdot)\right), u \otimes \psi(\cdot)\right\rangle .
$$

Since $f\left(s e_{1}+i(\cdot)\right) \in \mathcal{S}^{\prime}\left(\mathbb{R}^{m}\right) \subseteq \mathcal{S}^{\prime}\left(\mathbb{R}_{t} \times \mathbb{R}_{y^{\prime}}^{m-1}\right)$, it follows by the Schwartz kernel theorem that $\left\langle T_{s} \psi, u\right\rangle=\left\langle f\left(s e_{1}+i(\cdot)\right), u \otimes \psi(\cdot)\right\rangle$ defines for each $s \in \mathbb{R}, 0 \leq s \leq 1$, a linear map $T_{s}$ from $\mathcal{S}\left(\mathbb{R}_{y^{\prime}}^{m-1}\right)$ to $\mathcal{S}^{\prime}\left(\mathbb{R}_{t}\right)$ given by $T_{s} \psi=\langle f(s+i(\cdot), i(\cdot)), \psi(\cdot)\rangle=$ $f^{\psi}(s+i(\cdot)) \in \mathcal{S}^{\prime}\left(\mathbb{R}_{t}\right)$. Thus, for each $u \in \mathcal{S}\left(\mathbb{R}_{t}\right)$ we get

$$
\left\langle f_{j}^{\psi}(s+i \cdot), u(\cdot)\right\rangle \rightarrow\left\langle T_{s} \psi, u\right\rangle=\left\langle f^{\psi}(s+i(\cdot)), u(\cdot)\right\rangle .
$$

Lemma 4.2. $w \mapsto f^{\psi}(w)$ is holomorphic on the strip $0<\Re w<1$.

Proof. Consider the strip $S=\{w=s+i t \in \mathbb{C}: 0<\Re w<1\}$. It is enough to show that for every $\varphi \in C_{c}^{\infty}((0,1)), \eta \in C_{c}^{\infty}(\mathbb{R})$,

$$
\int_{\mathbb{R}^{2}} f_{j}^{\psi}(s+i t) \varphi(s) \eta(t) d s d t \rightarrow\left\langle f^{\psi}(s+i t), \varphi(s) \eta(t)\right\rangle .
$$

In fact, applying Fubini's theorem to

$$
\begin{aligned}
\int_{\mathbb{R}^{2}} f_{j}^{\psi}(s & +i t) \varphi(s) \eta(t) d s d t \\
& =\int_{\mathbb{R}^{2}} \varphi(s)\left[\int_{\mathbb{R}^{m-1}} f_{j}\left(s+i t, i y^{\prime}\right) \eta(t) \psi\left(y^{\prime}\right) d y^{\prime}\right] d s d t
\end{aligned}
$$

and recalling that $\operatorname{supp} \varphi \subseteq(0,1)$ we obtain

$$
\begin{aligned}
\int_{\mathbb{R}^{2}} f_{j}^{\psi}(s & +i t) \varphi(s) \eta(t) d s d t \\
& =\int_{0}^{1} \varphi(s)\left(\int_{\mathbb{R}^{m}} f_{j}\left(s e_{1}+i y\right)(\eta \otimes \psi)(y) d y\right) d s \\
& =\int_{0}^{1} \varphi(s) g_{j}(s) d s
\end{aligned}
$$

where $g_{j}(s)=\int_{\mathbb{R}^{m}} f_{j}\left(s e_{1}+i y\right)(\eta \otimes \psi)(y) d y$. Notice that if we fix $s \in[0,1]$ and let $j \rightarrow \infty$ we obtain

$$
\begin{aligned}
g_{j}(s) & \doteq \int_{\mathbb{R}^{m}} f_{j}\left(s e_{1}+i y\right)(\eta \otimes \psi)(y) d y \\
& \longrightarrow\left\langle f\left(s e_{1}+i \cdot\right),(\eta \otimes \psi)\right\rangle .
\end{aligned}
$$

Moreover, there exists a positive constant $C$ depending only on $\eta \otimes \psi$ such that

$$
\begin{aligned}
\left|g_{j}(s)\right| & =\left|\int_{\mathbb{R}^{m}} f_{j}\left(s e_{1}+i y\right)(\eta \otimes \psi)(y) d y\right| \\
& \leq C\left\|f_{j}\left(s e_{1}+i \cdot\right)\right\|_{h^{p}\left(\mathbb{R}^{m}\right)} \\
& \leq C s\left\|f_{j}\left(e_{1}+i \cdot\right)\right\|_{h^{p}}^{p}+C(1-s)\left\|f_{j}(0+i \cdot)\right\|_{h^{p}}^{p} \\
& \leq C \max _{x \in\left\{0, e_{1}\right\}} \sup _{j}\left\|f_{j}(x+i \cdot)\right\|_{h^{p}}^{p} \leq C^{\prime}<\infty .
\end{aligned}
$$

In view of (4.2) and (4.3), the dominated convergence theorem shows that (4.1) holds. 
Lemma 4.3. The holomorphic function

$$
f^{\psi}(s+i t)=\int_{\mathbb{R}^{m-1}} f\left(s+i t, i y^{\prime}\right) \psi\left(y^{\prime}\right) d y^{\prime}, \quad 0<s<1, t \in \mathbb{R},
$$

has weak boundary values at $s=0$ and at $s=1$ given by the $h^{p}$ distributions

$$
f^{\psi}(s+i t)=\int_{\mathbb{R}^{m-1}} f\left(s+i t, i y^{\prime}\right) \psi\left(y^{\prime}\right) d y^{\prime}, \quad s=0,1, t \in \mathbb{R} .
$$

Proof. All we need to show is that for every $\varphi \in C_{c}^{\infty}(\mathbb{R})$,

$$
\lim _{s \searrow 0} \int_{\mathbb{R}}\left(f^{\psi}(s+i t)-f^{\psi}(0+i t)\right) \varphi(t) d t=0
$$

and

$$
\lim _{s \nearrow 1} \int_{\mathbb{R}}\left(f^{\psi}(s+i t)-f^{\psi}(1+i t)\right) \varphi(t) d t=0 .
$$

To prove (4.4) notice that

$$
\begin{aligned}
\mid \int_{\mathbb{R}}\left(f^{\psi}(s+i t)\right. & \left.-f^{\psi}(0+i t)\right) \varphi(t) d t \mid \\
& =\left|\int_{\mathbb{R}^{m}}\left(f\left(s e_{1}+i y\right)-f(0+i y)\right)(\psi \otimes \varphi)(y) d y\right| \\
& \leq C_{\psi, \varphi}\left\|f\left(s e_{1}+i \cdot\right)-f(0+i \cdot)\right\|_{h^{p}} .
\end{aligned}
$$

We know from (2.14) that $\lim _{s \searrow 0}\left\|f\left(s e_{1}+i \cdot\right)-f(0+i \cdot)\right\|_{h^{p}}^{p}=0$, so letting $s \searrow 0$ in the last inequality we obtain

$$
\lim _{s \searrow 0} \int_{\mathbb{R}}\left(f^{\psi}(s+i t)-f^{\psi}(0+i t)\right) \varphi(t) d t=0 .
$$

The proof of (4.5) is analogous.

Returning to the function $f(x+i y), x \in[a, b] \subset \mathbb{R}^{m}, y \in \mathbb{R}^{m}$, and the associated function $f^{\psi}(s+i t), \psi \in \mathcal{S}\left(\mathbb{R}^{m-1}\right)$, Lemmas 4.1 , 4.2 and 4.3 may be summarized by saying that

(1) $w \mapsto f^{\psi}(w)$ is holomorphic on the strip $0<\Re w<r$;

(2) $f^{\psi}(s+i t)$ has weak boundary values at $s=0$ and at $s=r$ which are given by the $h^{p}$ distributions $t \mapsto f^{\psi}(0+i t)$ and $t \mapsto f^{\psi}(r+i t)$, respectively, with

$$
f^{\psi}(w)=\int_{\mathbb{R}^{m-1}} f\left(a+R\left(w, i y^{\prime}\right)\right) \psi\left(y^{\prime}\right) d y^{\prime} .
$$

It follows that (1) and (2) determine completely the function $f^{\psi}$ and, letting $\psi$ vary in $C_{c}^{\infty}\left(\mathbb{R}^{m-1}\right)$, this determines $f$ on

$$
\{(s+i t)(b-a), \quad 0<s<1\}+\Pi=[a, b]+i \mathbb{R}^{m} .
$$

We may rephrase (1) and (2) by saying that $f$ is a holomorphic function of $w$, $0<\Re w<r$, with values in $\mathcal{S}^{\prime}\left(\mathbb{R}^{m-1}\right)$. Thus, the function $f$ obtained in subsection 2.2 has the following uniqueness property: it is the only $\mathcal{S}^{\prime}\left(\mathbb{R}^{m-1}\right)$-valued extension that is holomorphic on the one-dimensional strip

$$
S(a, b)=\{a+(s+i t)(b-a), \quad 0<s<1, t \in \mathbb{R}\} \subset \mathbb{C}^{m}
$$

with boundary values given by the distributions $t \mapsto f(i t(b-a))$ and $t \mapsto$ $f((1+i t)(b-a))$. 


\section{Appendix A. The Approximation formula for tubes}

We will discuss the Baouendi-Treves approximation formula [BT] on an open subset $V \times \mathbb{R}^{m}$ of the tube $M=N \times \mathbb{R}^{m}$ where special coordinates have been chosen, as described in Section 1, so $\mathcal{V}^{\perp}$ is generated by the differential of the functions $Z_{k}(x, t)=x_{k}+i \phi_{k}(t)$. We will write $Z(x, t)=\left(Z_{1}(x, t), \ldots, Z_{m}(x, t)\right)$ and $\Phi(t)=\left(\phi_{1}(t), \ldots, \phi_{m}(t)\right)$. The local approximation operator applied to a null solution $f(x, t)$ is, for $\tau>0$,

$$
E_{\tau, h} f(x, t)=(\tau / \pi)^{m / 2} \int_{\mathbb{R}^{m}} e^{-\tau\left[Z(x, t)-Z\left(x^{\prime}, 0\right)\right]^{2}} f\left(x^{\prime}, 0\right) h\left(x^{\prime}\right) d x^{\prime} .
$$

Here $[\zeta]^{2} \doteq \zeta_{1}^{2}+\cdots+\zeta_{m}^{2}$ for $\zeta=\left(\zeta_{1}, \ldots, \zeta_{m}\right) \in \mathbb{C}^{m}$, which explains the meaning of $\left[Z(x, t)-Z\left(x^{\prime}, 0\right)\right]^{2}=\left[\left(x-x^{\prime}\right)+i \Phi(t)\right]^{2}$ in the formula. The function $h(x) \in C_{c}^{\infty}\left(\mathbb{R}^{m}\right)$ satisfies $h(x)=0$ for $|x| \geq 2$ and $h(x)=1$ in a neighborhood of $|x| \leq 1$. The integration with respect to $x^{\prime}$ must be understood as a duality bracket between the tempered distribution $f(\cdot, 0)$ and the test function $x^{\prime} \mapsto h\left(x^{\prime}\right) e^{-\tau\left[Z(x, t)-Z\left(x^{\prime}, 0\right)\right]^{2}}$ (note that $\left|e^{-\tau\left[Z(x, t)-Z\left(x^{\prime}, 0\right)\right]^{2}}\right|=e^{\tau|\Phi(t)|^{2}} e^{-\tau\left|x-x^{\prime}\right|^{2}}$ ). Since $f(\cdot, t)$ is, as an element of $L^{\infty}\left(N, \mathcal{S}^{\prime}\left(\mathbb{R}^{m}\right)\right)$, only defined up to a set of measure zero, we will have to check that $f(\cdot, 0)$ is well defined using the fact that $f(x, t)$ is a null solution of the structure. Fix $\psi \in \mathcal{S}\left(\mathbb{R}^{m}\right)$ and set $f_{\psi}(t) \doteq\langle f(\cdot, t), \psi(\cdot)\rangle$. Since we know that $f_{\psi}(t)$ is a bounded function defined a.e., if we prove that it is a locally Lipschitz function where it is defined it will have a continuous extension to all of $N$. We know that $\left\langle f, L_{j} \phi(y) \psi(x)\right\rangle=0, \phi \in C_{c}^{\infty}(N), \psi(x) \in \mathcal{S}\left(\mathbb{R}^{m}\right)$. Writing $\psi_{\ell}=\partial \psi / \partial x_{\ell}, 1 \leq \ell \leq m$, we have

$$
\int f_{\psi}(t) \frac{\partial \phi}{\partial t_{j}}(t) d t=\int\left(i f_{\psi_{j}}(t)-\sum_{k=1}^{m} \lambda_{j k}(t) f_{\psi_{k}}(t)\right) \phi(t) d t, \quad 1 \leq j \leq n
$$

The factor that multiplies $\phi(t)$ on the right hand side is essentially bounded on compact sets. Hence, the weak exterior derivative of $d_{t} f_{\psi}$ is in $L_{\mathrm{loc}}^{\infty}(N)$ and $f_{\psi}$ is locally Lipschitz by Rademacher's theorem. In particular, $f_{\psi}(0)$ is well defined for any $\psi \in \mathcal{S}\left(\mathbb{R}^{m}\right)$.

The modified approximation operator is

$$
G_{\tau, h} f(x, t)=(\tau / \pi)^{m / 2} \int_{\mathbb{R}^{m}} e^{-\tau\left[Z(x, t)-Z\left(x^{\prime}, t\right)\right]^{2}} f\left(x^{\prime}, t\right) h\left(x^{\prime}\right) d x^{\prime},
$$

and the remainder operator is

$$
G_{\tau, h} f(x, t)-E_{\tau, h} f(x, t)=R_{\tau, h} f(x, t) .
$$

An application of Stokes' theorem (see, e.g., [BCH, p.59]) shows that

$$
\begin{array}{rl}
R_{\tau, h} & f(x, t) \\
& =(\tau / \pi)^{m / 2} \sum_{j=1}^{n} \int_{\mathbb{R}^{m} \times[0, t]} e^{-\tau\left[Z(x, t)-Z\left(x^{\prime}, t^{\prime}\right)\right]^{2}} f\left(x^{\prime}, t^{\prime}\right) L_{j} h\left(x^{\prime}\right) d t_{j} \wedge d Z\left(x^{\prime}, t^{\prime}\right) .
\end{array}
$$


We now replace everywhere in the formulas for $G_{\tau, h}, E_{\tau, h}$ and $R_{\tau, h}, h\left(x^{\prime}\right)$ by $h\left(\varepsilon x^{\prime}\right)$ and let $\varepsilon \searrow 0$. Since $e^{-\tau\left[Z(x, t)-Z\left(x^{\prime}, t^{\prime}\right)\right]^{2}} L_{j}\left[h\left(\varepsilon x^{\prime}\right)\right] \rightarrow 0$ in $\mathcal{S}\left(\mathbb{R}^{m}\right)$, we obtain

$$
\begin{aligned}
G_{\tau} f(x, t)= & E_{\tau} f(x, t), \\
G_{\tau} f(x, t) & =(\tau / \pi)^{m / 2} \int_{\mathbb{R}^{m}} e^{-\tau\left[Z(x, t)-Z\left(x^{\prime}, t\right)\right]^{2}} f\left(x^{\prime}, t\right) d x^{\prime}, \\
E_{\tau} f(x, t) & =(\tau / \pi)^{m / 2} \int_{\mathbb{R}^{m}} e^{-\tau\left[Z(x, t)-Z\left(x^{\prime}, 0\right)\right]^{2}} f\left(x^{\prime}, 0\right) d x^{\prime} .
\end{aligned}
$$

At this point, we specialize the approximation formula to homogeneous solutions that belong to $L^{\infty}\left(N, h^{p}\left(\mathbb{R}^{m}\right)\right)$. We recall how the localizable Hardy spaces $h^{p}\left(\mathbb{R}^{m}\right)$, introduced by Goldberg in $[\mathrm{G}$, are defined. Fix once and for all a nonnegative function $\varphi \in \mathcal{S}\left(\mathbb{R}^{m}\right)$ with $\int_{\mathbb{R}^{m}} \varphi(x) d x=1$. For $u \in \mathcal{S}^{\prime}\left(\mathbb{R}^{m}\right)$ we define the small maximal function $m_{\varphi} u$ by

$$
m_{\varphi} u(x)=\sup _{0<t<1}\left|\left(u * \varphi_{t}\right)(x)\right|,
$$

where $\varphi_{t}(x)=t^{-m} \varphi(x / t)$.

Definition A.1. Let $0<p<\infty$. A tempered distribution $u \in \mathcal{S}^{\prime}\left(\mathbb{R}^{m}\right)$ belongs to $h^{p}\left(\mathbb{R}^{m}\right)$ if and only if $m_{\varphi} u \in L^{p}\left(\mathbb{R}^{m}\right)$, i.e.,

$$
\|u\|_{h^{p}} \doteq\left(\int_{\mathbb{R}^{m}}\left(m_{\varphi} u(x)\right)^{p} d x\right)^{1 / p}<\infty .
$$

For $p=\infty$, we set $h^{\infty}\left(\mathbb{R}^{m}\right)=L^{\infty}\left(\mathbb{R}^{m}\right)$.

For $0<p \leq 1$, the space $h^{p}\left(\mathbb{R}^{m}\right)$ is a complete metric space with the distance

$$
d(u, v)=\|u-v\|_{h^{p}}^{p}, \quad u, v \in h^{p}(\mathbb{R}) .
$$

For $p=1,\|u\|_{h^{1}}$ is a norm and $h^{1}\left(\mathbb{R}^{m}\right)$ is a normed space densely contained in $L^{1}\left(\mathbb{R}^{m}\right)$. For $p>1, h^{p}\left(\mathbb{R}^{m}\right)=L^{p}\left(\mathbb{R}^{m}\right)$ and $\|u\|_{h^{p}}$ is a norm equivalent to the usual $L^{p}$ norm.

If $f(x, t) \in L^{\infty}\left(N, h^{p}\left(\mathbb{R}^{m}\right)\right)$ is a homogeneous solution of the structure, we have that $f(\cdot, t) \in h^{p}\left(\mathbb{R}^{m}\right)$ for all $t \in N$ and

$$
\sup _{t \in N}\|f(\cdot, t)\|_{h^{p}}<\infty .
$$

Theorem A.1. Assume that $\mathcal{V}$ is a locally integrable tube structure on the tube $M=N \times \mathbb{R}^{m}$ of class $C^{k}, 1 \leq k \leq \infty$ and let $V \times \mathbb{R}^{m} \subset N \times \mathbb{R}^{m}$ be an open subset where appropriate coordinates and first integrals $Z_{1}(x, t), \ldots, Z_{m}(x, t)$ as described before exist. For any $f(t, x) \in L^{\infty}\left(N, h^{p}\left(\mathbb{R}^{m}\right)\right)$ that is a homogeneous solution of $\mathcal{V}$, set

$$
E_{\tau} f(x, t)=(\tau / \pi)^{m / 2} \int_{\mathbb{R}^{m}} e^{-\tau\left[Z(x, t)-Z\left(x^{\prime}, 0\right)\right]^{2}} f\left(x^{\prime}, 0\right) d x^{\prime} .
$$

Then, for every homogeneous solution $f(t, x) \in L^{\infty}\left(N, h^{p}\left(\mathbb{R}^{m}\right)\right), 0<p<\infty$,

$$
\begin{aligned}
& \left\|E_{\tau} f(\cdot, t)\right\|_{h^{p}} \leq C\|f(\cdot, t)\|_{h^{p}}, \\
& \lim _{\tau \rightarrow \infty}\left\|f(\cdot, t)-E_{\tau} f(\cdot, t)\right\|_{h^{p}}=0, \quad t \in V, \quad \tau>0 .
\end{aligned}
$$

Proof. The proof in the tube situation is simpler than previous known results ([HM], [BCH, p.78]) on the convergence of the Baouendi-Treves formula in local Hardy 
spaces, valid for general locally integrable structures. Consider the usual approximation of the identity associated to the Gaussian function

$$
g_{\varepsilon}(x)=\frac{1}{\varepsilon^{m}} g(x / \varepsilon), \quad g(x)=(1 / \pi)^{m / 2} \exp \left(-|x|^{2}\right) .
$$

Set $\psi_{t, s}=\varphi_{t} * g_{s}, 0<t, s \leq 1, \mathcal{B}=\left\{\psi_{1, s}: 0<s \leq 1\right\} \cup\left\{\psi_{t, 1}: 0<t \leq 1\right\}$ and

$$
m_{\mathcal{B}} u(x)=\sup _{\substack{0<\varepsilon \leq 1 \\ \psi \in \mathcal{B}}}\left|\left(u * \psi_{\varepsilon}\right)(x)\right|, \quad u(x) \in h^{p}\left(\mathbb{R}^{m}\right) .
$$

Since $\mathcal{B}$ is a bounded subset of $\mathcal{S}\left(\mathbb{R}^{m}\right),\left\|m_{\mathcal{B}} u\right\|_{L^{p}} \simeq\left\|m_{\varphi} u\right\|_{L^{p}}, u \in h^{p}\left(\mathbb{R}^{m}\right)$. If $h(x) \in h^{p}\left(\mathbb{R}^{m}\right)$ and $0<s, \varepsilon \leq 1$, then

$$
\left|\varphi_{s} * g_{\varepsilon} * h(x)\right|=\left|\psi_{t} * h(x)\right| \leq m_{\mathcal{B}} h(x)
$$

with $t=\max (s, \varepsilon)$ and $\psi \in \mathcal{B}$. Taking the sup in $0<s \leq 1$, raising the resulting inequality to the power $p$ and integrating with respect to $x$ we obtain

$$
\left\|g_{\varepsilon} * h\right\|_{h^{p}}=\left\|m_{\varphi}\left(g_{\varepsilon} * h\right)\right\|_{L^{p}} \leq\left\|m_{\mathcal{B}} h\right\|_{L^{p}} \leq C\left\|m_{\varphi} h\right\|_{L^{p}}=C\|h\|_{h^{p}} .
$$

For $h(x)=f(x, t)$ and $\varepsilon=\tau^{-1 / 2}$ this gives $\left\|G_{\tau} f(\cdot, t)\right\|_{h^{p}} \leq C\|f(\cdot, t)\|_{h^{p}}$, which proves (A.2) in view of (A.1). Furthermore, $g_{\varepsilon} * h \rightarrow h$ in $\mathcal{S}\left(\mathbb{R}^{m}\right)$ for $h \in \mathcal{S}\left(\mathbb{R}^{m}\right)$, which is a dense subspace of $h^{p}\left(\mathbb{R}^{m}\right)$, so (A.3) follows.

Set

$$
F_{\tau}(\zeta)=(\tau / \pi)^{m / 2} \int_{\mathbb{R}^{m}} e^{\tau\left[\zeta-i Z\left(x^{\prime}, 0\right)\right]^{2}} f\left(x^{\prime}, 0\right) d x^{\prime}, \quad \zeta \in \mathbb{C}^{m} .
$$

Notice that $x^{\prime} \mapsto e_{\zeta, \tau}\left(x^{\prime}\right) \doteq e^{\tau\left[\zeta-i Z\left(x^{\prime}, 0\right)\right]^{2}}$ is in $\mathcal{S}\left(\mathbb{R}^{m}\right)$ for any $\zeta \in \mathbb{C}^{m}$ and depends holomorphically on $\zeta$. Furthermore, the set

$$
X(R, \tau)=\left\{e_{\zeta, \tau}\left(x^{\prime}\right):|\Re \zeta| \leq R\right\} \subset \mathcal{S}\left(\mathbb{R}^{m}\right)
$$

is a bounded subset of $\mathcal{S}\left(\mathbb{R}^{m}\right)$ for $R, \tau>0$ fixed, as follows from $\left|e_{\zeta, \tau}\left(x^{\prime}\right)\right| \leq$ $e^{\tau R^{2}-\tau\left|\Im \zeta-x^{\prime}\right|^{2}} \leq C(\tau, R)$ as well as from similar estimates for the derivatives $D_{x^{\prime}}^{\alpha} e_{\zeta, \tau}\left(x^{\prime}\right), \alpha \in \mathbb{N}^{m}$. It follows that $F_{\tau}(\zeta), \tau>0$, is an entire function of $\zeta \in \mathbb{R}^{m}$ that remains bounded for $|\Re \zeta| \leq R, R>0$.

Corollary A.1. Let $f(t, x) \in L^{\infty}\left(N, h^{p}\left(\mathbb{R}^{m}\right)\right)$ be a homogeneous solution of $\mathcal{V}$. There exists a sequence $F_{j}(\zeta)$ of entire functions on $\mathbb{C}^{m}$, bounded for $\Re \zeta$ bounded, such that

(a) $\left\|F_{j}(i Z(\cdot, t))\right\|_{h^{p}} \leq\|f\|_{L^{\infty}\left(N, h^{p}\left(\mathbb{R}^{m}\right)\right)}, t \in V$;

(b) $\lim _{j \rightarrow \infty}\left\|F_{j}(i Z(\cdot, t))-f(\cdot, t)\right\|_{h^{p}}=0, t \in V$.

Corollary A.2. Let $f(t, x) \in L^{\infty}\left(N, h^{p}\left(\mathbb{R}^{m}\right)\right)$ be a homogeneous solution of $\mathcal{V}$ and set $\tilde{V}=-\Phi(V) \subset \mathbb{R}^{m}$. There exists a function $\tilde{f} \in L^{\infty}\left(\tilde{V}, h^{p}\left(\mathbb{R}^{m}\right)\right)$ such that

$$
f(x, t)=\tilde{f}(-\Phi(t)+i x), \quad t \in V, x \in \mathbb{R}^{m} .
$$

In particular, $\Phi\left(t_{1}\right)=\Phi\left(t_{2}\right)$ implies that $f\left(x, t_{1}\right)=f\left(x, t_{2}\right)$ as distributions in $\mathbb{R}^{m}$ and

$$
\|\tilde{f}\|_{L^{\infty}\left(\tilde{V}, h^{p}\left(\mathbb{R}^{m}\right)\right)}=\|f\|_{L^{\infty}\left(V, h^{p}\left(\mathbb{R}^{m}\right)\right)} \leq\|f\|_{L^{\infty}\left(N, h^{p}\left(\mathbb{R}^{m}\right)\right)} .
$$

Proof. Define $\tilde{f}(-\Phi(t)+i x)=\tilde{f}(i Z(x, t))=\lim _{j \rightarrow \infty} F_{j}(i Z(x, t))$. The claimed properties are easily checked. 
A.1. The globally integrable case. Let $N$ be a manifold of class $C^{k}, 1 \leq k \leq \infty$, and dimension $n$ and consider the tube manifold $M=N \times \mathbb{R}^{m}$.

Definition A.2. A complex vector subbundle $\mathcal{V}$ of $\mathbb{C} T M$, of rank $n$ and co-rank $m$, is a globally integrable tube structure of class $C^{k}$ if there exist $m$ real-valued functions $\phi_{k}: N \longrightarrow \mathbb{R}, k=1, \ldots, m$, of class $C^{k}$ such that the differentials of the functions

$$
Z_{k}(x, t)=x_{k}+i \phi_{k}(t), \quad k=1, \ldots, m, \quad x \in \mathbb{R}^{m}, t \in N,
$$

span $\mathcal{V}^{\perp}$ on $M=N \times \mathbb{R}^{m}$.

Example A.1. Let $N \subset \mathbb{R}^{m}$ be an embedding and let $\phi_{j}: N \rightarrow \mathbb{R}$ be the restriction to $N$ of the Cartesian coordinate functions $x_{j}, j=1, \ldots, m$. Then the standard tangential Cauchy-Riemann structure $\mathcal{V}$ on the tube CR submanifold $N+i \mathbb{R}^{m} \subset$ $\mathbb{C}^{m}$ is globally integrable and $\mathcal{V}^{\perp}$ is globally spanned by the differentials of the functions

$$
\begin{gathered}
Z_{k}(y, t)=y_{k}-i \phi_{k}(t)=-i\left(\left.x_{k}\right|_{N}+i y_{k}\right)=-\left.i z_{k}\right|_{M}, \\
k=1, \ldots, m, y \in \mathbb{R}^{m}, t \in N .
\end{gathered}
$$

Example A.2. The Mizohata structure in $\mathbb{R}^{2}$ spanned by the vector field

$$
\frac{\partial}{\partial t}-i t \frac{\partial}{\partial x}
$$

has the global first integral $Z(x, t)=x+i t^{2} / 2$.

Example A.3. Let $\Phi: \mathbb{R} \longrightarrow \mathbb{R}^{3}$ be an immersion such that $\Phi(\mathbb{R})$ is dense in a 2-torus $\mathbb{T}_{2} \subset \mathbb{R}^{3}$. Then $Z(x, t)=x+i \Phi(t)$ defines a globally integrable tube structure on $\mathbb{R} \times \mathbb{R}^{3}$ generated by a single vector field. This structure is isomorphic to the tube CR structure in $\mathbb{C}^{3}$ over the submanifold $\Phi(\mathbb{R})$, which is a self-winding orbit of a real vector field in $\mathbb{R}^{3}$ with a single minimal invariant set equal to $\mathbb{T}^{2}$.

Given a globally integrable tube structure on a connected tube manifold $M=$ $N \times \mathbb{R}^{m}$ with global first integral $Z(x, t)=x+i \Phi(t)=\left(Z_{1}(x, t), \ldots, Z_{m}(x, t)\right)$, $x \in \mathbb{R}^{m}, t \in N$, consider the set $\tilde{N}=-\Phi(N)$. Suppose without loss of generality that there is a distinguished point, $t_{0} \in N$ such that $\Phi\left(t_{0}\right)=0$. We have a global approximation formula for functions $f(t, x) \in L^{\infty}\left(N, h^{p}\left(\mathbb{R}^{m}\right)\right)$. Set

$$
\begin{aligned}
F_{\tau}(\zeta) & =(\tau / \pi)^{m / 2} \int_{\mathbb{R}^{m}} e^{\tau\left[\zeta-i Z\left(x^{\prime}, t_{0}\right)\right]^{2}} f\left(x^{\prime}, t_{0}\right) d x^{\prime} \\
& =(\tau / \pi)^{m / 2} \int_{\mathbb{R}^{m}} e^{\tau\left[\zeta-i x^{\prime}\right]^{2}} f\left(x^{\prime}, t_{0}\right) d x^{\prime} .
\end{aligned}
$$

For a point $i Z(x, t)=\xi+i \eta \in \tilde{N}$ we have the alternative representation

$$
F_{\tau}(\xi+i \eta)=(\tau / \pi)^{m / 2} \int_{\mathbb{R}^{m}} e^{\tau-\left[\eta-i x^{\prime}\right]^{2}} f\left(x^{\prime}, t\right) d x^{\prime},
$$

which follows from (A.1) for $(x, t)$ in a coordinate patch around $\left(x, t_{0}\right)$ and then for all $(x, t)$ by a connectedness argument. Then, taking any sequence $\tau_{j} \rightarrow \infty$ and applying Corollaries A.1 and A.2 in local charts of $N$ we obtain

Proposition A.1. Let $f(t, x) \in L^{\infty}\left(N, h^{p}\left(\mathbb{R}^{m}\right)\right), 0<p<\infty$, be a homogeneous solution of a globally integrable tube structure on a connected manifold $M=N \times \mathbb{R}^{m}$ 
and set $\tilde{N}=-\Phi(N)$. There exists a function $\tilde{f} \in L^{\infty}\left(\tilde{N}, h^{p}\left(\mathbb{R}^{m}\right)\right)$ and a sequence $\left(F_{j}(\zeta)\right)$ of entire functions on $\mathbb{C}^{m}$, bounded for $\Re \zeta$ bounded, such that

(1) the factorization $f=\tilde{f} \circ(i Z)$ holds, i.e.,

$$
f(x, t)=\tilde{f}(-\Phi(t)+i x), \quad t \in N, x \in \mathbb{R}^{m} ;
$$

(2) $\sup _{x \in \tilde{N}}\left\|F_{j}(x+i \cdot)\right\|_{h^{p}} \leq\|\tilde{f}\|_{L^{\infty}\left(\tilde{N}, h^{p}\left(\mathbb{R}^{m}\right)\right)}$;

(3) $\lim _{j \rightarrow \infty}\left\|F_{j}(x+i \cdot)-\tilde{f}(x, \cdot)\right\|_{h^{p}}=0, x \in \tilde{N}$.

\section{REFERENCES}

[BT] M. S. Baouendi and F. Treves, A property of the functions and distributions annihilated by a locally integrable system of complex vector fields, Ann. of Math. (2) 113 (1981), 387-421. MR607899 (82f:35057)

$[\mathrm{BCH}]$ S. Berhanu, P. Cordaro and J. Hounie, An Introduction to Involutive Structures, Cambridge University Press, 2008. MR2397326 (2009b:32048)

[Bo] S. Bochner, A theorem on analytic continuation of functions in several variables Ann. of Math. (2) 39 (1938), 14-19. MR 1503384

[BM] S. Bochner and W. T. Martin, Functions of several complex variables, Princeton University Press, 1948. MR0027863(10:366a)

[B1] A. Boggess, CR Manifolds and the Tangential Cauchy-Riemann Complex, Studies in Advanced Mathematics (1991), CRC Press. MR1211412 (94e:32035)

[B2] A. Boggess, The holomorphic extension of $H^{p}-C R$ functions on tube submanifolds, Proc. Amer. Math. Soc. 127 (1999), 1427-1435. MR1600104 (99h:32012)

[BD] Boivin and Dwilewicz, Extension and Approximation of CR Functions on Tube Manifolds, Trans. Amer. Math. Soc. 350 (1998), 1945-1956. MR.1443864 (98h:32011)

[Du] P. Duren, Theory of $H^{p}$ spaces, Academic Press (1970). MR0268655 (42:3552)

[G] D. Goldberg, A local version of real Hardy spaces, Duke Math. J., 46 (1979), 27-42. MR.523600 (80h:46052)

[HM] J. Hounie and P. Malagutti, On the convergence of the Baouendi-Treves approximation formula, Comm. P.D.E., 23 (1998), 1305-1347. MR1642611(2000b:32072)

[H] L. Hörmander, Notions of convexity, Birkhäuser (1994). MR1301332 (95k:00002)

Departamento de Matemática, Universidade Federal de São Carlos, São Carlos, SP, 13565-905, BRASIL

E-mail address: hoepfner@dm.ufscar.br

Departamento de Matemática, Universidade Federal de São Carlos, São Carlos, SP, 13565-905, BRASIL

E-mail address: hounie@dm.ufscar.br

Departamento de Matemática, Universidade Federal de São Carlos, São Carlos, SP, 13565-905, BRASIL

E-mail address: luis@dm.ufscar.br 